\title{
Aspects Clinique Et Paraclinique Des Paralysies Bilatérales Des Cordes Vocales
}

\author{
Do Santos Zounon Alexis, \\ Vodouhe Bidossessi Ulrich,
}

Unité d'ORL et Chirurgie Cervico-Faciale, Université Abomey-Calavi, Faculté des Sciences de la Santé de Cotonou, Bénin.

Aïssi Vanessa Monelle Benett Ablawa, Service d'ORL et Chirurgie Cervico-Faciale, Centre Hospitalier

Universitaire Mont-Godinne, commune d'Yvoir, Belgique

Adjibabi Wassi,

Yehouessi-Vignikin Bernadette,

Unité d'ORL et Chirurgie Cervico-Faciale, Université Abomey-Calavi,

Faculté des Sciences de la Santé de Cotonou, Bénin

Doi:10.19044/esj.2020.v16n15p212 URL:http://dx.doi.org/10.19044/esj.2020.v16n15p212

\section{Résumé}

L'immobilité bilatérale des cordes vocales peut mettre en jeu le pronostic vital du patient. L'objectif de l'étude a été d'analyser les éléments diagnostiques à évaluer avant la prise en charge. Il s'est agi d'une étude transversale et descriptive portant sur les cas d'immobilité bilatérale des cordes vocales reçus entre $1^{\text {er }}$ Janvier 2008 et le $1^{\text {er }}$ Mars 2018. Les données socio-démographiques, cliniques, paracliniques et les étiologies ont été recueillies. En dix ans 56 patients ont été reçus et examinés. L'âge moyen a été de 56 ans avec une sex-ratio de 1,07. Les principaux signes répertoriés étaient la dyspnée (44 cas ; 78,6\%), la dysphonie ( 8 cas ; 14,3\%) et les fausses routes $(2$ cas ; 3,6\%). Les cordes vocales étaient immobiles en adduction paramédiane ( 29 cas ; 51,79\%), en adduction médiane ( 25 cas ; 44,64\%) ou en abduction ( 2 cas ; 3,6\%). Les deux principales causes retrouvées ont été l'intubation prolongée et la thyroïdectomie totale. La paralysie des cordes vocales est peu fréquente. La nasofibroscopie est la clé du diagnostic. L'intubation prolongée et la chirurgie thyroïdienne en sont les causes les plus fréquentes.

Mots clés : Paralysie Laryngée Bilatérale, Cordes Vocales, Diagnostic 


\title{
Clinical and Paraclinical Aspects of Bilateral Vocal Cord Paralysis
}

\author{
Do Santos Zounon Alexis, \\ Vodouhe Bidossessi Ulrich,
}

Unité d'ORL et Chirurgie Cervico-Faciale, Université Abomey-Calavi, Faculté des Sciences de la Santé de Cotonou, Bénin.

Aïssi Vanessa Monelle Benett Ablawa, Service d'ORL et Chirurgie Cervico-Faciale, Centre Hospitalier

Universitaire Mont-Godinne, commune d'Yvoir, Belgique

Adjibabi Wassi,

Yehouessi-Vignikin Bernadette,

Unité d'ORL et Chirurgie Cervico-Faciale, Université Abomey-Calavi,

Faculté des Sciences de la Santé de Cotonou, Bénin

\begin{abstract}
Bilateral immobility of the vocal cords can be life-threatening. The aim of the study was to analyze the diagnostic elements to be assessed before treatment. This was a cross-sectional and descriptive study on the cases of bilateral immobility of the vocal cords received between January 1, 2008 and March 1, 2018. Socio-demographic, clinical, paraclinical and etiology data were collected. In ten years 56 patients have been received and examined. The average age was 56 with a sex ratio of 1.07 . The main signs listed were dyspnea (44 cases; 78.6\%), dysphonia ( 8 cases; $14.3 \%$ ) and false routes (2 cases; $3.6 \%)$. The vocal cords were immobile in paramedian adduction (29 cases; $51.79 \%$ ), in median adduction (25 cases; $44.64 \%$ ) or in abduction (2 cases; $3.6 \%$ ). The two main causes found were prolonged intubation and total thyroidectomy. Vocal cord paralysis is uncommon. Nasofibroscopy is the key to diagnosis. Prolonged intubation and thyroid surgery are the most common causes.
\end{abstract}

Keywords: Bilateral Laryngeal Paralysis, Vocal Cords, Diagnosis.

\section{Introduction}

Les cordes vocales, au nombre de deux, constituent un élément essentiel du larynx dont elles définissent l'étage glottique. Petites structures fibro-musculaires, elles sont situées en arrière du cartilage thyrö̈de. Elles s'écartent pendant la respiration, se rapprochent pendant la déglutition et 
vibrent l'une contre l'autre pendant la phonation. L'immobilité bilatérale des cordes vocales selon leur position peut se manifester par une dyspnée mettant en jeu le pronostic vital, une dysphonie ou des troubles de la déglutition (Ozedemir, 2013). Il s'agit d'une affection rare mais difficile à gérer en raison de la morbidité importante qu'elle peut générer (Brake, 2015 ; Djafarou, 2017). L'évaluation des voies aériennes supérieures commence par un interrogatoire et un examen physique incluant une nasofibroscopie. La présente étude fait le point des éléments diagnostiques à analyser avant la prise en charge.

\section{Méthode}

Il s'est agi d'une étude transversale de type descriptif qui s'est déroulée dans le service d'ORL au Centre Hospitalier Universitaire Mont-Godinne dans la commune d'Yvoir en Belgique. L'étude a concerné les dossiers des patients présentant des cas d'immobilité bilatérale des cordes vocales reçus entre $1^{\text {er }}$ Janvier 2008 et le $1^{\text {er }}$ Mars 2018. Les dossiers ont été exploités dans le strict anonymat.

Une fiche d'enquête établie pour la circonstance a permis de recenser les données socio-démographiques, cliniques, paracliniques et les étiologies (Intubation, thyroïdectomie totale, traumatisme laryngé, paralysie recurrentielle centrale, radiothérapie et/ou chimiothérapie, autres). Les données ont été recueillies dans le dossier des patients et traitées par le logiciel Microsoft Excel Office 365. L'élaboration des graphiques et l'analyse des variables ont été faites par le logiciel $\mathrm{R}$ version 3.5.1.

\section{Résultats}

\section{Fréquence}

L'enquête a concerné 56 patients, soit en moyenne une incidence de 5 nouveaux cas par an. La figure 1 indique la répartition annuelle du recrutement des patients.

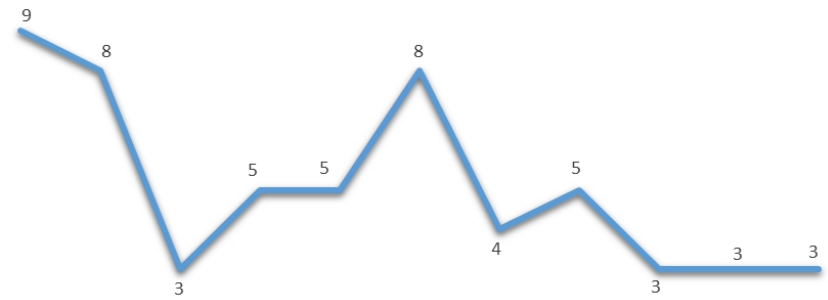

$\begin{array}{lllllllllll}2008 & 2009 & 2010 & 2011 & 2012 & 2013 & 2014 & 2015 & 2016 & 2017 & 2018\end{array}$

Figure 1: Répartition des malades selon l'année de recrutemen 


\section{Aspects socio-démographiques}

Parmi les 56 patients inclus dans l'étude, 29 (51,8\%) étaient de sexe masculin et 27 (48,2\%) étaient de sexe féminin soit une sex-ratio de 1,07.

L'âge moyen était de $56 \pm 18$ ans avec une variation allant de 14 à 89 ans. L'âge médian était de 59 ans. La figure 2 indique la répartition des patients par tranches d'âges.

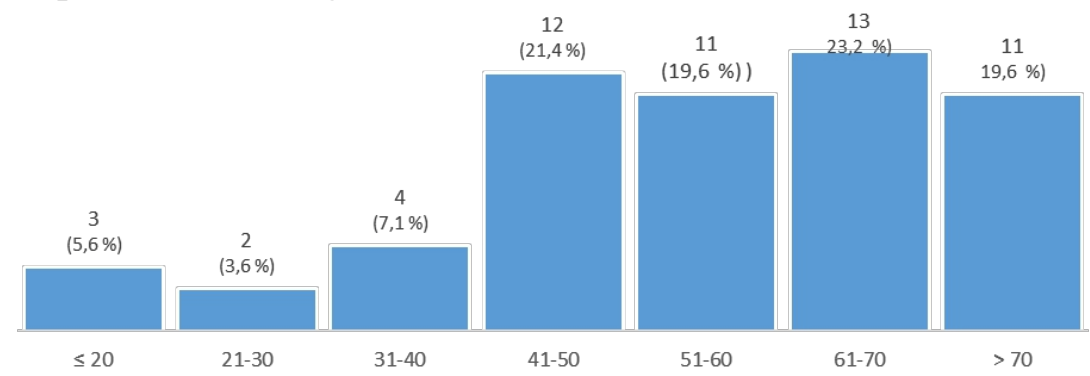

Figure 2: Répartition selon l'âge des patients suivis pour immobilité bilatérale des cordes vocales, $n=56$.

\section{Aspects cliniques}

\section{Antécédents médicaux}

Les cancers des voies aéro-digestives supérieures ont été signalés chez sept patients. Ces patients ont bénéficié de radio-chimiothérapie (3 cas), radiothérapie exclusive (3cas) et chimiothérapie exclusive (1 cas). Les autres antécédents médicaux recensés étaient l'œsophagite ou la gastrite ( 7 cas) et le pyrosis (2 cas).

\section{Antécédents chirurgicaux}

Onze (11) patients $(19,6 \%)$ n'ont signalé aucun antécédent chirurgical. La Figure 3 illustre les différentes interventions chirurgicales antérieures.

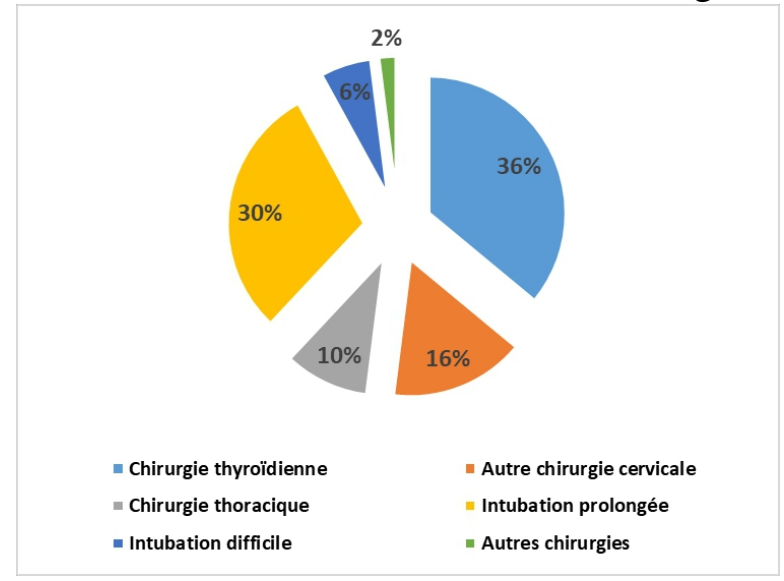

Figure 3 : Répartition des patients selon les antécédents chirurgicaux 


\section{Enquête sociale}

Le tabagique a été noté chez 36 patients $(64,3 \%)$ et non précisé chez 20 patients $(35,7 \%)$. Le Tableau I indique la distribution des 36 patients tabagiques selon le sexe et la quantité fumée.

Tableau I: Répartition des cas selon le nombre de paquet-années et le sexe

\begin{tabular}{ccccc}
\hline & \multicolumn{3}{c}{ Effectif } & Proportion (\%) \\
\cline { 2 - 4 } & Masculin & Féminin & Total & \\
\hline Non tabagique & 11 & 12 & 23 & 63,9 \\
< 1 paquet-année & 2 & 1 & 3 & 8,3 \\
$\geq 1$ paquet-année & 4 & 0 & 4 & 11,1 \\
Tabagique ancien & 4 & 2 & 6 & 16,7 \\
Total & 21 & 15 & 36 & 100,0 \\
\hline
\end{tabular}

\section{Signes fonctionnels}

Le tableau II indique les différents signes fonctionnels enregistrés et leurs proportions.

\begin{tabular}{ccc}
\multicolumn{3}{c}{ Tableau II: Répartition des malades selon le symptôme majeur de consultation } \\
\hline & Effectif $(\mathrm{n})$ & Proportion $(\%)$ \\
\hline Dyspnée & 44 & 78,6 \\
Dysphonie & 8 & 14,3 \\
Dysphagie & 0 & 0 \\
Fausse route & 2 & 3,6 \\
Dyspnée + Dysphonie & 1 & 1,8 \\
Aucune plainte majeure & 1 & 1,8 \\
Total & 56 & 100 \\
\hline
\end{tabular}

\section{Nasofibroscopie}

L'immobilité des cordes vocales a été complète chez 45 patients $(80,4 \%)$ et partielle chez 11 patients $(19,6 \%)$. Les cordes vocales étaient en adduction paramédiane chez 29 patients $(51,8 \%)$, en adduction médiane chez 25 patients $(44,6 \%)$ et en abduction chez 2 patients $(3,6 \%)$. Le Tableau III présente la relation entre le symptôme majeur et la position des cordes vocales.

Tableau III: Répartition des patients selon le symptôme majeur et la position des cordes

\begin{tabular}{ccccc} 
& \multicolumn{3}{c}{ Pocales } & Total \\
\cline { 2 - 4 } & $\begin{array}{c}\text { Adductio } \\
\mathrm{n}\end{array}$ & $\begin{array}{c}\text { Adduction } \\
\text { Paramédian }\end{array}$ & $\begin{array}{c}\text { Abductio } \\
\mathrm{n}\end{array}$ & \\
\hline Dyspnée & 23 & $\mathrm{e}$ & 0 & \\
Dysphonie & 0 & 6 & 2 & 84 \\
Canule & 1 & 1 & 0 & 2 \\
Dyspnée + Dysphonie & 0 & 1 & 0 & 1 \\
Aucune plainte majeure & 1 & 0 & 0 & 1 \\
Total & 25 & 29 & 2 & 56 \\
\hline
\end{tabular}


Les figures 4 et 5 montrent les positions des cordes vocales en adduction et en abduction respectivement.

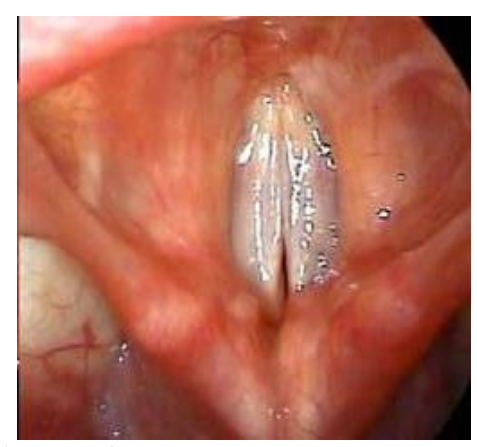

Figure 4 : Paralysie bilatérale des cordes vocales en adduction

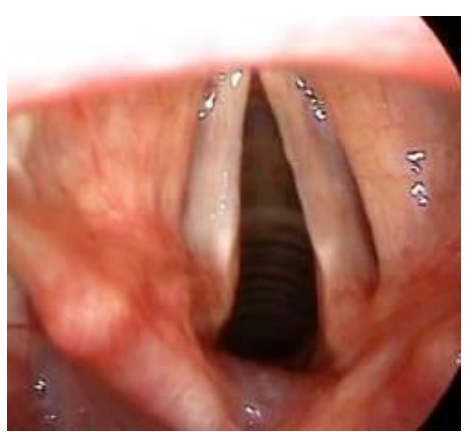

Figure 5 : Paralysie bilatérale des cordes vocales en abduction

Les autres lésions objectivées à la nasofibroscopie étaient un granulome laryngé ( 5 cas ; 8,9\%), une synéchie des cordes vocales ( 3 cas ; 5,4\%), un œdème des cordes vocales et/ou laryngé $(2$ cas $; 3,6)$, et un cas de sténose sousglottique post-traumatique $(1,8 \%)$.

\section{Aspect paraclinique}

\section{Electromyographie laryngée (EMGL)}

L'EMGL a été réalisée chez 24 patients. Elle a objectivé un potentiel de re-inervation chez 7 patients $(29,2 \%)$ et un potentiel de dénervation chez 4 patients $(16,7 \%)$. Un patient a présenté simultanément des potentiels de dénervation à droite et des potentiels de réinnervation à gauche.

\section{Radio-vidéo-déglutition}

Elle a noté deux cas de pénétration laryngée et un cas de fausse route primaire.

\section{Causes}

Le Tableau IV résume les différentes causes identifiées et leurs proportions.

Tableau IV: Répartition des malades selon l'étiologie

\begin{tabular}{ccc}
\hline & Effectif & Proportion (\%) \\
\hline Post-intubation prolongée & 14 & 25 \\
Post-thyroïdectomie totale & 13 & 23,2 \\
Indéterminé & 7 & 12,5 \\
Post-intubation & 6 & 10,7 \\
Post-traumatique & 5 & 8,9 \\
Paralysie récurrentielle d'origine centrale & 2 & 3,6 \\
Post Radiothérapie & 2 & 3,6 \\
Post Radio et Chimiothérapie & 2 & 3,6 \\
Contexte tumoral & 1 & 1,8
\end{tabular}


Post chirurgie cardiaque

Post chirurgie thoracique

Polyneuropathie motrice périphérique

Paralysie sur malade de Lyme

Total

$\begin{array}{cc}1 & 1,8 \\ 1 & 1,8 \\ 1 & 1,8 \\ 1 & 1,8 \\ 56 & 100,0\end{array}$

Le tableau $\mathbf{V}$ présente la relation entre l'étiologie et le positionnement des cordes vocales.

Tableau V: Relation entre l'étiologie et le positionnement des CV

\begin{tabular}{|c|c|c|c|c|}
\hline & \multicolumn{3}{|c|}{ Positionnement des CV } & \multirow{2}{*}{$\begin{array}{c}\text { Tota } \\
1\end{array}$} \\
\hline & Adduction & $\begin{array}{c}\text { Adduction } \\
\text { Paramédian } \\
\mathrm{e} \\
\end{array}$ & Abduction & \\
\hline Post-traumatique & 1 & 4 & 0 & 5 \\
\hline $\begin{array}{c}\text { Paralysie récurrentielle } \\
\text { Centrale }\end{array}$ & 0 & 2 & 0 & 2 \\
\hline Post-thyroïdectomie totale & 5 & 7 & 1 & 13 \\
\hline Post-intubation & 2 & 4 & 0 & 6 \\
\hline Post-intubation prolongée & 8 & 6 & 0 & 14 \\
\hline Contexte tumoral & 1 & 0 & 0 & 1 \\
\hline Post Radio et Chimiothérapie & 2 & 0 & 0 & 2 \\
\hline Post Radiothérapie & 1 & 1 & 0 & 2 \\
\hline Post chirurgie cardiaque & 0 & 1 & 0 & 1 \\
\hline Post chirurgie thoracique & 0 & 1 & 0 & 1 \\
\hline $\begin{array}{l}\text { Polyneuropathie motrice } \\
\text { périphérique }\end{array}$ & 0 & 1 & 0 & 1 \\
\hline Paralysie sur maladie de Lyme & 0 & 0 & 1 & 1 \\
\hline Indéterminé & 5 & 2 & 0 & 7 \\
\hline Total & 25 & 29 & 2 & 56 \\
\hline
\end{tabular}

\section{Discussion}

\section{Aspects socio-demographiques}

Dans cette étude l'incidence annuelle de l'immobilité bilatérale des cordes vocales est de 5 nouveaux cas. Ce taux rejoint celui de Misiolek, Ziora, Namyslowski (2007) ainsi que Brake et al (2015) au Canada qui avaient publié respectivement 6 et 4 cas par an. Par contre Omari (2017) au Maroc a rapporté une incidence annuelle moyenne supérieure soit 9 nouveaux cas par an. Le sexe masculin était le plus représenté dans la présente enquête $(51,8 \%)$. Cette prédominance masculine a été aussi constatée par Min, Hoong, Kim (2008) qui ont noté 59,4\% de sujet de sexe masculin dans leur série. Toutefois Omari (2017) a remarqué une nette prédominance féminine $(91,89 \%)$. La participation féminine est aussi mise en évidence par plusieurs autres auteurs africains, elle pourrait s'expliquer par le fait que la thyroïdectomie est beaucoup plus réalisée chez les femmes par rapport aux hommes (Vignikin- 
Yêhouéssi, 2008; Do Santos Zounon, 2019; Leye, 2016; Koumare, 2016; Ille, 2017 ; Poumale, 2017). L'âge moyen de notre échantillon a été de 56 ans similaire à celui d'autres travaux (Misiolek, 2007; Omari, 2017; Gaudon, 2005).

\section{Aspect clinique}

\section{Signes fonctionnels :}

Les immobilités bilatérales des cordes vocales ont des conséquences fonctionnelles variables. La dyspnée est associée aux atteintes bilatérales en fermeture. Une atteinte bilatérale en ouverture peut entraîner une dysphonie et peut s'accompagner de fausses routes. L'interrogatoire recherche de manière systématique des troubles de la déglutition liés à l'atteinte du nerf pneumogastrique ainsi que divers signes associés en cas de lésions d'autres paires crâniennes (Lacau, 2002). Dans la présente étude la symptomatologie est dominée par la dyspnée (78,6\%). Le constat était identique chez d'autres auteurs (Omari, 2017; Lacau, 2002; Murty, 1989). Cette insuffisance respiratoire pourrait s'expliquer par la réduction de la filière aérienne au cours de la respiration du fait d'une immobilité des cordes vocales.

\section{Positionnement des cordes vocales :}

La nasofibroscopie est l'examen de choix pour la mise en évidence de la paralysie des cordes vocales (Murty, 1989; Boumendil, 2016). Elle a été le seul moyen diagnostique dans la présente recherche. Elle est connue pour sa bonne tolérance surtout si le patient est informé des désagréments inhérents à sa réalisation (Fomai, 2019). Dans notre étude, 96,4\% des patients présentaient des cordes vocales en adduction (médiane ou paramédiane). Cette position des cordes vocales justifierait la dyspnée conséquente. Les paralysies en abduction étaient rares.

\section{Electromyographie}

L'électromyographie laryngée (EMGL) des cordes vocales a permis d'établir le degré d'atteinte nerveuse chez 7 patients. Elle se pratique par un opérateur entraîné, sous anesthésie locale par voie transcutanée du fait du risque permanent de décompensation respiratoire (Lacau, 2002). L'EMGL affirme l'origine neurogène de l'immobilité laryngée, faisant la distinction entre une paralysie laryngée et une arthrite cricoaryténoïdienne (Frachet, 1992). Elle permet aussi d'établir la topographie lésionnelle et le pronostic (Remacle, 2006). La présence d'un potentiel d'action indique un bon pronostic (Laccourreye, 2003). 


\section{Données étiologiques}

Les principales causes de paralysie bilatérale des cordes vocales dans cette étude étaient l'intubation prolongée et la thyroïdectomie totale. Ce constat rejoint celui Lacau (2002) en France. Pour Tucker (1980) de même que Thakhar et al (2008), la chirurgie thyrö̈dienne était la principale cause de paralysie bilatérale des cordes vocales. Par contre, Terris et al (1992), a retrouvé comme étiologie dominante les cancers pulmonaires (55\%), suivie des diplégies laryngées post-thyrö̈dectomie dans $25 \%$ des cas. Des causes idiopathiques ont été mentionnées dans certains travaux (Hammami, 2011; Gupta, 2013; Chen, 2007; Huppler, 1955). Des taux plus bas ont été notés par Omari (2017) avec 5,4\%. Par contre Gupta J. et al (2013) ont trouvé une incidence plus élevé de paralysies idiopathiques (25\%). Les étiologies idiopathiques, par définition, n’ont pas de cause évidente. L'adéquation du bilan détermine également l'incidence des causes idiopathiques. Cela peut expliquer l'incidence élevée d'étiologie idiopathique dans certaines séries. L'imagerie améliorée et l'endoscopie par fibre optique ont permis de réduire l'incidence de la «paralysie idiopathique» de 29 à 11\% (Chen, 2007; Huppler, 1955).

\section{Conclusion}

L'immobilité bilatérale des cordes vocales est une affection peu fréquente pouvant mettre en jeu le pronostic vital. Son diagnostic positif se fait à la nasofibroscopie qui est l'examen de référence. La manifestation clinique est fonction de la position des cordes vocales qui peuvent être en adduction, en position paramédiane ou plus rarement en abduction. L'intubation prolongée et la chirurgie thyrö̈dienne sont les étiologies les plus fréquentes.

\section{References:}

1. Ozdemir S, Tuncer U, Tarkan O, Kara K, Surmelioglu O. Carbon dioxide laser endoscopic posterior cordotomy technique for bilateral abductor vocal cord paralysis: a 15-year experience. JAMA Otolaryngol Head Neck Surg. 2013; 139: 401-4.

2. Brake K Maria and Anderson Jennifer. Bilateral vocal fold immobility: a 13 year review of etiologies, management and the utility of the empey index. Brake and Anderson Journal of Otolaryngology - Head and Neck Surgery 2015; 44: 27. DOI 10.1186/s40463-015-0080-8

3. Djafarou AC, Ndiaye C, Ndiaye M, Diom ES, Ahmed H, Thiam NF, Pilor N, Maiga S, Diouf MS, Deguenonvo REA, Tall A, Diallo BK, Ndiaye IC, Diouf R. Paralysie récurrentielle bilatérale : 1'aryténoïdopexie est-elle toujours compétitive? Médecine d'Afrique Noire 2017; 6409: 443-448 
4. Misiolek M, Ziora D, Namyslowski G. Long term results in patients after combined laser total arytenoidectomy with posterior cordectomy for bilateral vocal cord paralysis. Eur Arch Otolaryngol 2007; 264: 895-900.

5. Omari M. Les paralysies laryngées [Thèse : Med]. Fès : F.M.P ; 2017 $\mathrm{N}^{\circ} 026,188 \mathrm{p}$.

6. Min JY, Hoong SD, Kim K. Long term results of artecoll injection laryngoplasty for patients with unilateral vocal fold motion impairment. Arch Otolaryngol Head Neck Surg 2008; 134 (5):490-496.

7. Vignikin-Yêhouéssi B, Flatin M, Vodouhè S, Hounkpè Y, Médji A. Place de la thyroïdectomie en pratique ORL au CNHU de Cotonou. Sciences et Médecine. Rev. CAMES - Série A, Vol. 06,2008.

8. Do Santos Zounon Alexis ; Attolou Gilles ; Vodouhe Ulrich Bidossessi ; Adjibabi Wassi ; Yehouessi-Vignikin Bernadette. Particularité de prise en charge des goitres plurinodulaires bénins à l'Hôpital d'Instruction des Armées de Cotonou, Bénin. Médecine d'Afrique Noire 2019 ; 66(7) : 370378.

9. Leye A ; Ndiaye N ; Leye Ym. Les thyrö̈dectomies au Centre Hospitalier National de Pikine-Dakar (CHNP) : Indications et résultats chez 417 patients. Revue africaine de Médecine interne 2016 ; 3 : 36-40.

10. Koumare S ; Soumare L ; Sacko O ; Camara M. Prise en charge des goitres en chirurgie A du CHU du point G. Mali Médical 2016 ; 29 : 13-17.

11. Ille S ; James Didier L ; Saidou A. Résultats de cinq ans de thyrö̈dectomie au service d'ORL et de Chirurgie Cervico-Facial de l'Hopital National de Niamey (Niger). European Scientific Journal 2017 ; 13 : 44-52.

12. Poumale F; Doui $\mathrm{AD}$; Nghario L. La chirurgie thyroïdienne à Bangui : Indications et suites opératoires à propos de 135 cas. Health Sciences and Diseases 2017 ; 18 : 39-43.

13. Gaudon L, Lawson G, Remacle M. Subtotal carbon dioxide laser arytenoidectomy for the treatment of bilateral vocal fold immobility: longterm results. Ann Otol Rhinol Laryngol. 2005; 114(2):115-21.

14. Lacau J, Chevalier D, Arnoux-sondt B, Beutter P. Paralysies récurrentielles de l'adulte. SFORL. L O b Conseil - Recommandations pour la pratique clinique - Octobre $2002: 44 p$

15. Murty G, Smith M. Reccurent Laryngeal nerv palsy following Heart-lung transplantation: three cases of vocal cord augmentation in the acute phase. J laryngol Otol 1989; 103: 986-969.

16. Boumendil I. Laser CO2 et microchirurgie endolaryngée [Thèse : Med]. Rabat : F.M.P ; 2016 N$^{\circ} 109$.

17. Fomai W, Amanai B, Lawson SLA, Pegbessoui E, Boko E, Kpemissi E. Tolérance et vécu de la nasofibroscopie chez les patients au CHU Sylvanus Olympio de Lomé : Evaluation par auto-questionnaire. J. TUN ORL 2019; 42: 16-19. 
18. Frachet B. Pratique phoniatrique en Orl. Paris : Masson, 19921 :6-244.

19. Remacle M, Lawson G. Paralysies laryngées. EMC (Elsevier SAS, Paris), Oto-rhino-laryngologie, 20-675-A-10, 2006.

20. LACCOURREYE O. Tiré à part sur Paralysies laryngées unilatérales 2003 ; édition SANTOR 8p.

21. Tucker HM. Cord vocal paralysis, etiology and management, laryngoscope 1980; 90:585-590.

22. Thakkar K., Gerber ME. Endoscopic posterieur costal cartilage graft placement for acute management of pediatric bilateral vocal fold paralysis without tracheostomy. Internat Journal of Ped Otolaryngol 2008; 72: 1555 58.

23. Terris D, Arneistein DP, Neguyen HH. Comtenporary evaluation of vocal cord paralysis, Otolaryngol head neck surg 1992;107:84-90.

24. Hammami B, Kallel S, Kolsi N, Smaoui L, Chakroun A, Charfeddine I, Ghorbel A. Traitement des diplégies laryngées en fermeture: apport du laser. J. tun ORL-n ${ }^{\circ} 26$ Juin-décembre 2011: 37- 40.

25. Gupta J, Varshney S, Bist S, Bhagat S. Clinico-Etiolological Study of Vocal Cord Paralysis. Indian J Otolaryngol Head Neck Surg. 2013 Jan; 65(1): 16-19.

26. Chen HC, Jen YM, Wang CH, Lee JC, Lin YS. Aetiology of vocal cord paralysis. ORL J Otorhinolaryngol Relat Spec. 2007;69:167-171. doi: 10.1159/000099226.

27. Huppler EG. Causes of vocal cord paralysis. Proc Staff Meet Mayo Clin. 1955;30:578-579. 